\title{
Uncovering the Mechanism of Ge-Gen-Qin-Lian Decoction for Treating Ulcerative Colitis Based on Network Pharmacology and Molecular Docking Verification
}

\section{Lin Xu}

China Academy of Chinese Medical Sciences https://orcid.org/0000-0003-2487-6528

Jiaqi Zhang

China Academy of Chinese Medical Sciences Xiyuan Hospital

\section{Zedan Zhang}

China Academy of Chinese Medical Sciences

\section{Yifan Wang}

Department of Gastroenterology, Peking University Traditional Chinese Medicine Clinical Medical School (Xiyuan)

\section{Fengyun Wang}

China Academy of Chinese Medical Sciences Xiyuan Hospital

\section{Xudong Tang ( $\nabla$ txdly@sina.com )}

China Academy of Chinese Medical Sciences, Dongcheng District, 100700 Beijing, China https://orcid.org/0000-0003-0391-3895

\section{Research}

Keywords: Ulcerative colitis, Network pharmacology, molecular docking, Traditional Chinese Medicine, GeGen-Qin-Lian decoction

Posted Date: September 17th, 2020

DOl: https://doi.org/10.21203/rs.3.rs-74864/v1

License: (c) (i) This work is licensed under a Creative Commons Attribution 4.0 International License.

Read Full License

Version of Record: A version of this preprint was published at Bioscience Reports on February 1st, 2021. See the published version at https://doi.org/10.1042/BSR20203565. 


\section{Abstract}

Background and objective: Ge-Gen-Qin-Lian Decoction (GGQLD), a traditional Chinese medicine (TCM) formula, has been widely used for ulcerative colitis (UC) in China while the pharmacological mechanisms still remain unclear. The present research was designed to clarify the underlying mechanism of GGQD against UC.

Methods: In this research, a GGQLD-compound-target-UC (G-U) network was constructed based on public databases to clarify the relationship between active compounds in GGQLD and potential targets. GO and KEGG pathway enrichment analyses were performed to investigate biological functions associated with potential targets. A protein-protein interaction network was constructed to screen and evaluate hub genes and key active ingredients, another GO and KEGG pathway analyses were subsequently performed on hub genes. Molecular docking was used to verify the activities of binding between hub targets and ingredients.

Results: Finally, 83 potential therapeutic targets and 118 correspond active ingredients were obtained by network pharmacology. GO and KEGG enrichment analysis revealed that GGQLD had an effect of antiinflammation, antioxidation, and immunomodulatory. The effect of GGQLD on UC might be achieved by regulating the balance of cytokines (eg., IL6, TNF, IL1 13, CXCL8, CCL2, IL10, IL4, IL2) in immune system and inflammation-related pathways, such as IL-17 pathway and Th17 cell differentiation pathway. Besides, molecular docking results demonstrated that the main active ingredients, quercetin, exhibited good affinity to hub targets.

Conclusion: This research fully reflects the characteristics of multi-component and multi-target for GGQLD in the treatment of UC. Furthermore, the present study provided new insight into the mechanisms of GGQLD against UC.

\section{Introduction}

Ulcerative Colitis (UC) is an idiopathic chronic inflammatory bowel disease (IBD), characterized by persistent inflammation of the entire large intestine, which causes abdominal pain, bloody diarrhea, and fecal urgency[1]. The incidence of UC is increasing in the 21 st century, especially in Asia[2]. While the etiology of UC is still incompletely elucidated, it is thought to be associated with innate and adaptive immunity, gut barrier function, and pathogen sensing and response[3, 4]. Treatment of UC mainly includes medical management and colectomy. Current therapies are limited by increased risks of infection of cancer by loss of clinical benefit. Since the dissatisfaction with conventional medication, complementary and alternative therapies are commonly used in the patient with $U C[5,6]$, including traditional Chinese medicine (TCM), dietary supplements, probiotics, and mind/body interventions $[7,8]$. Among them, the therapeutic efficacy and safety of several TCM formulas in IBD have been shown in some double-blind randomized controlled trials[9, 10]. 
Ge-Gen-Qin-Lian decoction (GGQLD), a well-known TCM formula, was first described in Shang Han Lun written by Zhongjing Zhang[11], has more than 2,000 years of clinical application history in China. GGQLD is composed of 4 herbs: Gegen (Puerariae lobatae Radix), Huangqin (Scutellariae Radix), Huanglian (Coptidis Rhizoma) and Gancao (Glycyrrhizae Radix), at the ratio of 5:3:3:2[12]. In clinic, GGQLD has been commonly used for the treatment of diarrhea[13]. Recently, a meta-analysis included 22 trials involving 2028 patients with UC showed that GGQLD significantly improved the clinical effectiveness and recurrence rate compared with western medicine[14]. However, despite the known therapeutic effects of GGQLD, its pharmacological and molecular mechanisms of action have not been fully elucidated.

Network pharmacology is a part of bioinformatics, integrating bioinformatics and system medicine. Since network pharmacology can reflect the characteristics of multi-component and multi-target of TCM, it has been widely used in TCM research and advance drug discovery in recent years $[15,16]$. This provides a novel way for clarification of mechanisms of GGQLD that exert therapeutic effects in UC. In this study, we attempted to undertake a network pharmacology study to unveil the crucial genes and pathways that are involved in the pathogenesis of GGQLD against UC (see Fig. 1). These results may provide a new target for the treatment of GGQLD against UC.

\section{Materials And Methods}

\section{Data preparation}

\section{Construction of GGQLD chemical ingredients database}

All chemical ingredients of each herb in GGQLD were obtained from Traditional Chinese Medicine Systems Pharmacology Database and Analysis Platform (TCMSP) (https://tcmspw.com/tcmsp.php)[17]. TCMSP is a unique systems pharmacology platform, which integrated a large scale structural data (29,384 chemicals in total with 13,144 unique molecules) with manually curated information for all registered herbs in Chinese pharmacopeia [18]. It can also provide ADME-related properties data, like human oral bioavailability (OB), half-life (HL), drug-likeness (DL), Caco-2 permeability (Caco-2), bloodbrain barrier (BBB) and Lipinski's rule of five (MW, AlogP, TPSA, Hdon, Hacc)[19].

\subsubsection{Drug screening and evaluation}

Drug screening and evaluation in GGQLD were mainly identified by OB and DL administered by TCMSP. $\mathrm{OB}$ and $\mathrm{DL}$ are two important indicators in ADME. DL is a qualitative concept used in drug design for how "druglike" a substance is concerning factors like bioavailability, helps to optimize pharmacokinetic and pharmaceutical properties, such as solubility and chemical stability[20]. OB is the fraction of an oral administered drug that reaches systemic circulation, which reveals the convergence of the ADME process. High oral bioavailability is often a key indicator to determine the drug-like property of bioactive molecules as therapeutic agents[21]. Then, according to recommendations from TCMSP, we established preset criteria of $\mathrm{OB} \geq 30 \%$ and $\mathrm{DL} \geq 0.18$ to screen for the possible bioactive ingredients in GGQLD. 


\section{GGQLD-related Target prediction}

Corresponding protein targets of each active ingredient in GGQLD were predicted using TCMSP. Predicted targets from TCMSP were extracted and converted to gene names using UniProtKB (http://www.uniprot.org). Target information was set to Homo sapiens and aggregated for further analysis.

\section{Uc Related Genes Database Construction}

UC-related genes were retrieved from the DisGeNET database (https://www.disgenet.org/), Genecards database (https://www.genecards.org/), and DrugBank database (https://www.drugbank.ca/). The DisGenNET is a knowledge management platform integrating and standardizing data about diseaseassociated genes and variants from multiple sources. It also covers the full spectrum of human diseases as well as normal and abnormal traits[22, 23]. The GeneCards database is a one-stop-shop for searchable human gene annotations[24, 25]. DrugBank is a web-enabled database containing comprehensive molecular information about drugs, their mechanisms, their interactions, and their targets[26]. DrugBank was searched for known UC drugs/drug-like compounds which were approved by the United States Food and Drug Administration (FDA) for clinical trials. And finally, the common targets shared by at least two databases were retained as UC-related targets, while the other targets were removed.

\section{Construction Of A Ggqld-compound-target-uc Network For Ggqld}

Intersections of GGQLD targets and UC-related targets were regarded as potential targets of GGQLD for the treatment of UC. The corresponding chemical compound of the intersect targets were thought to be possible therapeutic components that affect UC. The potential targets were obtained by Venn. A GGQLDcompound-target-UC (G-U) network was constructed to clarify the relationship between active compounds in GGQLD and potential targets. This network was constructed and visualized using Cytoscape 3.7.2 software.

\section{Comprehensive Analysis}

\section{Functional enrichment analysis of GGQLD related targets}

To investigate biological functions associated with potential targets, we performed Gene Ontology (G0) and Kyoto Encyclopedia of Genes and Genomes (KEGG) pathway enrichment analyses via the "clusterProfiler" R package. "clusterProfiler" R package serves as a user-friendly enrichment tool with integrated gene cluster analysis based on multiple resources[27]. GO enrichment analysis was used to explain and annotate genes by three dimensions, including cellular component (CC), molecular function 
(MF), and biological process (BP) analyses. The KEGG database was mainly used for pathway analysis. Besides, at least three genes contained and $P$-values below 0.05 were considered statistically significant.

\section{Protein-protein Interaction Network Between Targets In The C-dts Network}

Protein-protein interaction (PPI) is of pivotal importance in the regulation of biological systems and is consequently implicated in the development of disease states[28]. The potential targets were introduced into the STRING database (https://string-db.org/) to perform PPI analysis. STRING, an online PPI analysis database, currently features the largest number of organisms (5090) and proteins (24.6 million), has very broad and diverse, benchmarked data sources[29]. The species was limited to "Homo sapiens", and the minimum required interaction score was set to be high confidence (0.700). The PPI network was visualized with Cytoscape 3.7.2 software.

\section{Hub Gene And Key Active Compounds Screening And Evaluation}

To further find out the hub genes in the potential targets, the top 10 hub genes were identified using MCC methods in "cytoHubba" of Cytoscape 3.7.2 software[30]. Furthermore, GO and KEGG pathway analyses of hub genes were performed via Database for Annotation, Visualization and Integrated Discovery (DAVID) (https://david.ncifcrf.gov/), a web-based analysis tool. Gene counts > 3 and a p-value below 0.05 were considered as the cutoff criterion. To reveal the relationship between hub genes and possible therapeutic components, the corresponding chemical compound of hub genes were incorporated into a Sankey diagram.

\section{Verification Through Molecular Docking}

Molecular docking was used to predict the activities of binding of proteins to compounds via CB-Dock (http://cao.labshare.cn/cb-dock/). CB-Dock is a protein-ligand docking method that automatically identifies the binding sites, calculates the center and size, customizes the docking box size according to the query ligands, and then perform the molecular docking with AutoDock Vina[31]. The PDB formats of proteins were derived from the RCSB PDB database (https://www.rcsb.org/), and SDF formats of ligands were obtained from PubChem database (https://pubchem.ncbi.nlm.nih.gov/). respectively. The style of ligand and receptor were set as "Ball\&stick" and "Cartoon", respectively. The color of ligand and receptor were set as "By Element" and "By Chain", respectively. The vina sore represents a certain binding activity between a ligand and a protein. The lower the vina score, the more stable the ligand binds to the protein.

\section{Results}




\section{Bioactive Compounds in GGQLD}

Bioactive ingredients in GGQLD and corresponding ADME information were extracted from the TCMSP data server. In total, 154 bioactive compounds were identified in GGQLD. After filter by drug-like threshold ( $O B \geq 30 \%$ and $D L \geq 0.18$ ), a total of 139 ingredients were identified, including 14 components of Huanglian, 35 components of Huangqin, 4 components of Gegen, 92 components of Gancao. Some compounds are shared in two or more herbs, such as quercetin (MOL000098) are shared in Huanglian and Gancao, beta-sitosterol (MOL000358) are shared in Gegen and Huangqin, coptisine (MOL001458) and epiberberine (MOL002897) are shared in Huanglian and Huangqin, formononetin (MOL000392) is shared in Gegen and Gancao. Detailed information regarding these ingredients is shown in Table S1.

\section{Targets Of Ggqld}

Potential targets in GGQLD were predicted in TCMSP. In total, 259 targets were identified for 139 bioactive compounds (4 compounds did not receive predictions), Afterwards, predicted targets from TCMSP were extracted and converted to gene names using UniProtKB. From the result, we can see that many compounds were predicted to target the same proteins, such as there are 62 ingredients target to PPARG.

\section{Uc Related Targets}

Identification of UC related targets was searched using the keyword "Ulcerative colitis" from the following resources: DisGeNET database, Genecards database, and DrugBank database. A total of 5273 genes were searched, including 914 genes from the DisGeNET database, 4356 genes selected from GeneCards, 17 FDA-approved compounds for the treatment of UC, and 64 genes identified from the DrugBank database. As a result, there are 776 common targets shared by at least two databases, which were retained as UC-related targets.

\section{Construction Of A Ggqld-compound-target-uc Network}

Based on the GGQLD target database and UC-related target database, 83 overlap genes were identified as the potential targets for GGQLD against UC, as shown in the Venn diagram (Fig. 2). The result showed that 10 genes are overlapping with the four databases (GGQLD, DisGenet, GeneCards, DrugBank), including ABCG2, CD40LG, CYP3A4, IFNG, MPO, NOS2, NR1I2, PPARG, PTGS1, and PTGS2. Detailed information regarding these targets is shown in Table $\mathrm{S} 2$.

There were 118 active ingredients correspond to the 83 potential targets. To better understand the complex relationships between all bioactive compounds in GGQLD and their UC-related targets, a G-U network was constructed (Fig. 3). There were 205 nodes in the G-U network composed of 118 active ingredients and $83 \mathrm{UC}$ targets, connected by 866 interactions. Besides, as shown in Fig. 3, node size 
indicated the number of connections calculated using "NetworkAnalyzer," an analytical tool in Cytoscape 3.7.2. The details of the G-U network were listed in Table S3.

\section{GO and KEGG pathway enrichment analysis of the C-DT Network}

To further validate whether biological functions enriched by candidate targets as mentioned above were correlated with UC, GO and KEGG enrichment analysis was performed via R. Three GO terms were analyzed, which included CC, MF, and BP. The top $10 \mathrm{GO}$ enrichment results for each term are shown in Fig. 4. In the CC group, the GO terms mainly included the "vesicle lumen", "secretory granule lumen", and "membrane raft". In the MF group, the GO terms were mainly involved "cytokine receptor binding", "heme binding", and "receptor ligand activity". Moreover, BP results indicated that GGQLD may regulate UC related biological processes, such as "reactive oxygen species metabolic process," "response to lipopolysaccharide," "response to reactive oxygen species" and "response to oxidative stress".

Additionally, 123 KEGG pathways were found. The KEGG enrichment analysis showed that the 83 potential targets were closely related to several critical pathways associated with UC, such as "IL-17 signaling pathway", "TNF signaling pathway," "Th17 cell differentiation", "Inflammatory bowel disease", "HIF-1 signaling pathway" and "Inflammatory bowel disease". The top 20 enriched KEGG signaling pathways were shown in Fig. 4. These findings demonstrated that the regulation of inflammation and immunity may be the main mechanism of GGQLD in the treatment of UC. The detailed GO and KEGG pathway enrichment analysis result was listed in Table S4.

\section{Ppi Network Analysis}

To further investigate the relationship between the 83 overlap genes, PPI was analyzed using the STRING online database, high confidence target proteins interaction data with a score $>0.7$ were selected. The obtained PPI network file was imported into the Cytoscape 3.7.2 software for visualizing. We found 77 nodes and 609 interactions in the PPI network, and the results are shown in Fig. 5.

\section{Hub Genes Investigation And Screening Of Key Active Ingredients}

According to the PPI network, the top 10 core nodes were identified, ranked by MCC in the "cytoHubba" plugin. Thus, 10 genes were identified as hub genes: IL6, TNF, STAT3, IL1ß, CXCL8, CCL2, ICAM1, IL10, IL4, IL2, as shown in Fig. 6-A. Also, another GO and KEGG analyses were conducted on the 10 hub genes to validate their biological functions. The enrichment analysis results validated that the 10 hub genes were strongly related to UC. For instance, IL6 is involved in "immune response", "cellular response to lipopolysaccharide", "Inflammatory bowel disease", all of which are closely related to UC. The top 5 GO terms and KEGG pathways of the 10 hub genes were shown in Fig. 6-B. The detail GO and KEGG enrichment of the 10 hub genes were listed in table S5. Moreover, a Sankey diagram was used to show 
the corresponding chemical compound that acts on hub genes, as shown in Fig. 6-C. Six ingredients in GGQLD correspond to hub genes were identified, including quercetin, formononetin, kaempferol, licochalcone a, oroxylin a, and wogonin. From the Sankey diagram, we can see that quercetin, shared in Huanglian and Gancao, targets most of the hub genes.

\section{Verification With Molecular Docking}

To further validate potential targets in UC, we performed molecular docking on quercetin with the 10 hub genes (IL6, TNF, STAT3, IL1 $\beta$, CXCL8, CCL2, ICAM1, IL10, IL4, IL2). Docking analysis successfully predicted vina scores between quercetin and the 10 hub genes, which were all negative and less than -6 , the results were listed in Table 1. Particularly, molecular docking between quercetin and TNF has the highest cavity size and the lowest Vina score. Overall, molecular docking results indicated that quercetin had good binding activities to the 10 hub genes, as shown in Fig. 7.

Table 1

The results of Cavity-detection guided Blind Docking.

\begin{tabular}{|c|c|c|c|c|c|c|c|c|c|}
\hline \multirow[t]{2}{*}{ ligand } & \multirow[t]{2}{*}{ protein } & \multirow[t]{2}{*}{ Vina score } & \multirow[t]{2}{*}{ Cavity size } & \multicolumn{3}{|c|}{ Center } & \multicolumn{3}{|c|}{ Size } \\
\hline & & & & $x$ & $y$ & z & $x$ & $y$ & $z$ \\
\hline \multirow[t]{10}{*}{ Quercetin } & IL6 & -7.2 & 551 & 4 & -2 & 10 & 27 & 21 & 21 \\
\hline & TNF & -9.1 & 863 & 8 & 63 & 31 & 21 & 21 & 21 \\
\hline & STAT3 & -8 & 527 & 16 & 12 & 18 & 21 & 21 & 21 \\
\hline & IL-1 & -6.7 & 197 & 40 & 9 & 56 & 21 & 21 & 21 \\
\hline & CXCL8 & -6.3 & 185 & -15 & 22 & 24 & 21 & 21 & 21 \\
\hline & CCL2 & -6.3 & 48 & 31 & 30 & 16 & 21 & 21 & 21 \\
\hline & ICAM1 & -6.6 & 100 & 50 & 92 & -12 & 21 & 21 & 21 \\
\hline & IL10 & -6.6 & 485 & 11 & 26 & 17 & 21 & 21 & 21 \\
\hline & IL4 & -7.3 & 105 & 82 & 35 & 11 & 21 & 21 & 21 \\
\hline & IL2 & -7.7 & 615 & 32 & 49 & 35 & 21 & 21 & 21 \\
\hline
\end{tabular}

\section{Discussion}

Nowadays, TCM is being more frequently chosen by patients to treat UC, and many TCM formulas have been successfully utilized for the treatment of UC[32, 33], including GGQLD. Vivo and Vitro studies have provided multiple lines of evidence indicating that some ingredients of GGQLD, such as berberine and Glabridin, have definite beneficial effects against UC[14]. Given the particularity of multi-component, multi-target, and multichannel of TCM formulae, the underlying molecular mechanisms triggered by 
GGQLD in the treatment of UC is far from being clarified. New approaches are now being considered for drug-target exploration and identification of potential active ingredients in TCM research, including network pharmacology[34]. Here, we used network pharmacology technology to predict the putative mechanism involved within the therapeutic effect of GGQLD in UC. In this study, we identified 139 bioactive compounds and 259 targets from the 4 herbs in GGQLD. Furthermore, 83 potential therapeutic targets and 118 correspond active ingredients were identified, and a G-U network was established with 205 nodes and 866 interactions. Also, GO and KEGG enrichment was further enriched based on the 83 potential target genes and a PPI network was constructed.

BP enrichment results indicated that a large number of genes were associated with oxidative stress, a key etiological factor of UC, including "reactive oxygen species metabolic process", "response to reactive oxygen species", "response to oxidative stress", "regulation of reactive oxygen species metabolic process", "cellular response to oxidative stress", et al. The gastrointestinal tract is prone to reactive oxygen species (ROS) attack. Alterations of the balance between ROS production and the capacity to rapidly detoxify reactive intermediates lead to oxidative stress, which is an essential factor in the pathogenesis of gastrointestinal mucosal disease[35]. During inflammatory episodes, neutrophils and macrophages infiltrate intestinal mucosa at the sites of IBD and release large amounts of ROS and cytokines including interleukin (IL)-1 $\beta$, IL-6, and tumor necrosis factor (TNF)-a. Excessive levels of ROS released by the inflamed stroma elicit oxidative damage to DNA[36], proteins[37], and lipids, ultimately, promote the initiation and progression of UC. Response to lipopolysaccharide (LPS) was also enriched in $\mathrm{BP}$, which also could play a pathogenic role in UC[38]. LPS is a major component of the outer membrane of Gram-negative bacteria[39], plays a key role in host-pathogen interactions with the innate immune system[40] and development of inflammatory diseases[41]. LPS impairs intestinal barrier function by causing inhibition of intestinal restitution and stimulates the release of proinflammatory cytokines. Therefore, our result indicates that GGQLD may also have a role in regulating intestinal microbiota.

Additionally, the KEGG result indicates that most of the disease-related pathways are about immune and inflammatory, tumor-related signaling pathways, and virus infection-related signaling pathways, most of them have been reported to be closely related to UC. For example, IL-17, a key mediator in the pathogenesis of intestinal inflammation, up-regulated in inflamed mucosa from UC patients. The disease severity in UC patients is also correlated with the IL-17 level in peripheral blood mononuclear cells[42]. Besides, IL-17 is produced mainly by T helper 17 (Th17) cells and other sources including natural killer cells, mast cells, and neutrophils. Th17 cells are considered to be key effector T cells of UC pathophysiology. New data from mouse models of IBD suggest that T cell plasticity, particularly along the Th1-Th17 and Th17-Treg axes, plays an important role in the regulation of intestinal immune responses[43]. Our functional enrichment analysis results indicated that anti-inflammation, antioxidation, and immunomodulatory may be the mechanism of GGQLD against UC.

Therefore, we further constructed a PPI network to unraveling the complex molecular relationships underline the potential targets, and 10 hub genes were identified by "cytohubba", including IL6, TNF, STAT3, IL1 $\beta$, CXCL8, CCL2, ICAM1, IL10, IL4, and IL2. Most of them are cytokines, such as 
proinflammatory cytokines (IL6, TNF IL1 $\beta$, CXCL8, CCL2), and anti-inflammatory cytokines (IL10, IL4, IL2). Cytokines are structurally diverse proteins with profound functional relevance to the maintenance of physiological homeostasis, including chemokines, interferons, interleukins, lymphokines, and tumor necrosis factors[44]. Cytokines are involved in intestinal homeostasis and pathological processes associated with IBD [45]. For example, M1 macrophages promote colonic inflammation via the production of the pro-inflammatory cytokine IL-6, CCL2, and TNF-a[46]. IL6, a key proinflammatory cytokine in the pathogenesis of multiple inflammatory diseases, acts on mesenchymal and epithelial cells to induce the recruitment of polymorphonuclear leukocytes (PMNs) and macrophages essential for wound healing. Recently, In a genome-wide meta-analysis of 20,550 patients with CD, 17,647 patients with UC, and more than 40,000 individuals without IBD (controls) result indicate that therapeutics designed to block IL6R signaling might be effective in the treatment of IBD[47]. Chemokines are small secreted proteins that orchestrate migration and positioning of immune cells within the tissues[48]. During innate and adaptive responses, chemokines are essential for the function of the immune system. CXCL8 is one of the first and most intensively studied chemokines acting as a pro-inflammatory chemokine[49]. Patients with UC have elevated levels of CXCL8 and CCL2 in colonic mucosa compared to healthy volunteers[50]. TNF is another key cytokine in IBD pathology, induces intestinal epithelial cell apoptosis in the context of IBD and murine disease models[51]. Furthermore, treatment strategies targeting TNF signaling are administered systemically and efficacious in UC, such as infliximab, adalimumab, and golimumab.

Cytokines are crucial for the maintenance of immune system. For example, IL-10 is a key immunosuppressive cytokine expressed by many cell types, particularly important in maintaining the intestinal microbe-immune homeostasis. Polymorphisms in the IL-10 locus confer risk for UC, deficient in either IL-10 or IL-10 receptor exhibit severe intestinal inflammation and marked pro-inflammatory cytokines secretion in mice and human[52]. IL-10 production by Th17 cells has been strongly related to the acquisition of regulatory properties by Th17 cells and the resolution of intestinal inflammation[53]. IL4, the core signature of Th2 responses, induces differentiation of naive helper T cells to Th2 cells[54]. Enormous reports have also provided pieces of evidence that IL-4 participates in the pathogenesis of $\operatorname{IBD}[55,56]$. Human IL-4-treated regulatory macrophages promote epithelial wound repair, reduce cytokineinduced epithelial barrier defects, and are beneficial in a murine model of acute colitis[57]. IL-2 is known as a $T$ cell growth factor[58], negatively regulate immune-mediated inflammation and stimulate tissue repair processes[59]. low-dose IL-2 has therapeutic effects on DSS-induced colitis and potential clinical value in treating $U C[60]$.

Cytokine signaling pathways involving transcription factors of the signal transducers and activators of transcription (STAT) family play a key role in the pathogenesis of IBD[44]. STAT3 activation occurs as a result of cytokine binding (for example, IL6), several studies have reported an increased expression of STAT3 or STAT3 phosphorylation in human IBD [61, 62]. STAT3 has also been shown to be critical in modulating the balance of Th17 and regulatory T (Treg) cells, as well as in promoting CD4(+) T cell proliferation[63]. Intracellular adhesion molecule-1 (ICAM-1), is a transmembrane glycoprotein of the immunoglobulin family, constitutively expressed on vascular endothelial cells and upregulated in 
inflamed colonic tissue[64]. It has been demonstrated that ICAM-1 expression increased in colonic lysates from UC patients. ICAM-1 can also be up-regulated in response to proinflammatory stimuli, such as TNF$\alpha$, IL-1 $\beta$, and IFN- $\gamma$. Particularly, ICAM-1 signaling seems to produce the recruitment of inflammatory immune cells[65] such as macrophages[66] and granulocytes[67]. Meanwhile, the GO and KEGG analysis of the 10 hub nodes showed that a considerable number of genes were involved in pathways including "Inflammatory bowel disease", and "Cytokine-cytokine receptor interaction".

Sankey diagram indicated that quercetin corresponds to most of the hub targets. Quercetin is a plantderived polyphenolic compound belonging to the flavonols (a subclass of flavonoids) and has shown beneficial effects in the prevention and/or treatment of several pathological conditions, due to its antioxidant, anti-inflammatory, anti-fibrotic, antimicrobial, and antitumoral activities[68-70]. In gastrointestinal tract, quercitrin can reduct the early stage inflammation by reducing IL-1 $\beta$, IL- 6 , TNF- $a$ levels, and NF-KB expression[70]. Various evidence has shown the anti-inflammatory activity of Quercitrin in experimental colitis[71, 72]. Moreover, modulation of intestinal microbiota is another way for quercetin to exert a therapeutic effect. Recent studies showed that quercetin reshapes intestinal microbiota in several diseases, such as obesity, Non-alcoholic fatty liver disease, and atherosclerosis, suggesting a prebiotic effect of the flavonol[73, 74]. Furthermore, molecular docking was conducted between quercetin and the 10 hub genes to verify the binding activities between active ingredients and potential targets. The results showed that quercetin had good binding activities to IL6, TNF, STAT3, IL1 $\beta$, CXCL8, CCL2, ICAM1, IL10, IL4, and IL2.

Cytokines that either promote or suppress intestinal inflammation has led to some efficacious therapeutics for IBD [75]. In general, these therapies can be grouped into two categories: blockade of proinflammatory or enhancement of anti-inflammatory cytokine pathways[76]. The discovery and characterization of these therapies indicate that simultaneous regulation of anti-inflammatory factors and pro-inflammatory factors may become a more potential treatment strategy for IBD. Our research suggests that GGQLD may have such a therapeutic effect.

\section{Conclusions}

Current research based on network pharmacology could provide a novel and systematic analysis way for the research of Chinese herbal formula. However, it should be noted that there were some limitations in our article. On the one hand, focusing on validated target genes may exclude potential targets that have not been validated by the experiment. On the other hand, there is a lack of verification analysis in our research. Thus, further clinical and basic research is needed to validate our results and to elucidate the molecular mechanism in GGQLD.

Overall, the present study suggests that quercetin may be the main active ingredient in GGQLD. Cytokines (eg., IL6, TNF, IL1 $\beta$, CXCL8, CCL2, IL10, IL4, IL2) might be the potential therapeutic targets of GGQLD in UC. Furthermore, the effect of GGQLD on UC might be achieved by regulating the balance of cytokines in immune system and inflammation-related pathways, such as IL-17 pathway and Th17 cell differentiation 
pathway. Last but not least, this research showed that network pharmacology is a powerful tool for identifying active compounds and potential targets derived from TCM.

\section{Abbreviations}

biological process (BP); blood-brain barrier (BBB); Caco-2 permeability (Caco-2)

cellular component (CC); Database for Annotation, Visualization and Integrated Discovery (DAVID); druglikeness (DL); Food and Drug Administration (FDA); Ge-Gen-Qin-Lian Decoction (GGQLD); Gene Ontology (GO); GGQLD-compound-target-UC (G-U); half-life (HL); interleukin (IL); Intracellular adhesion molecule-1 (ICAM-1); Kyoto Encyclopedia of Genes and Genomes (KEGG); lipopolysaccharide (LPS); molecular function (MF); oral bioavailability (OB); polymorphonuclear leukocytes (PMNs); Protein-protein interaction (PPI); reactive oxygen species (ROS); regulatory $\mathrm{T}$ (Treg); signal transducers and activators of transcription (STAT); T helper 17 (Th17); Traditional Chinese Medicine Systems Pharmacology Database and Analysis Platform (TCMSP); tumor necrosis factor (TNF); ulcerative colitis (UC)

\section{Declarations}

\section{Acknowledgements}

Not applicable

\section{Author contributions}

Lin Xu, Feng-Yun Wang and Xu-Dong Tang designed the study. Jia-Qi Zhang, Ze-Dan Zhang and Yi-Fan Wang screened and extracted the data. Lin Xu and Jia-Qi Zhang conducted the data analysis. Lin Xu wrote the paper. Feng-Yun Wang and Xu-Dong Tang reviewed and edited the manuscript. All authors have read and agree to the published version of the manuscript.

\section{Funding Statement}

This work has been financially supported by the National Natural Science Foundation of China (No. 81830118); the National Natural Science Foundation of China (No. 81774303).

\section{Data Availability}

The data used to support the result of this study can be obtained from the corresponding author.

\section{Ethics approval and consent to participate}

Not applicable.

\section{Consent for publication}


Not applicable.

\section{Conflicts of Interest}

The authors declare that there is no conflict of interest.

\section{References}

1. Ungaro R, Mehandru S, Allen PB, et al. Ulcerative colitis. Lancet. 2017;389:1756-70.

2. $\mathrm{Ng} \mathrm{SC}$, Shi HY, Hamidi N, et al. Worldwide incidence and prevalence of inflammatory bowel disease in the 21 st century: a systematic review of population-based studies. Lancet. 2018;390:2769-78.

3. Pickard JM, Zeng MY, Caruso R, et al. Gut microbiota: Role in pathogen colonization, immune responses, and inflammatory disease. Immunol Rev. 2017;279:70-89.

4. Graham DB, Xavier RJ. Pathway paradigms revealed from the genetics of inflammatory bowel disease. Nature. 2020;578:527-39.

5. Heuschkel R, Afzal N, Wuerth A, et al. Complementary medicine use in children and young adults with inflammatory bowel disease. Am J Gastroenterol. 2002;97:382-8.

6. Rawsthorne P, Clara I, Graff LA, et al. The Manitoba Inflammatory Bowel Disease Cohort Study: a prospective longitudinal evaluation of the use of complementary and alternative medicine services and products. Gut. 2012;61:521-7.

7. Picardo S, Altuwaijri M, Devlin SM, et al. Complementary and alternative medications in the management of inflammatory bowel disease. Therap Adv Gastroenterol. 2020;13:1756284820927550.

8. Langhorst J, Wulfert $\mathrm{H}$, Lauche R, et al. Systematic review of complementary and alternative medicine treatments in inflammatory bowel diseases. J Crohns Colitis. 2015;9:86-106.

9. Holleran G, Scaldaferri F, Gasbarrini A, et al. Herbal medicinal products for inflammatory bowel disease: A focus on those assessed in double-blind randomised controlled trials. Phytother Res. 2020;34:77-93.

10. Currò D, laniro G, Pecere $S$, et al. Probiotics, fibre and herbal medicinal products for functional and inflammatory bowel disorders. Br J Pharmacol. 2017;174:1426-49.

11. Liu T, Tian X, Li Z, et al. Metabolic profiling of Gegenqinlian decoction in rat plasma, urine, bile and feces after oral administration by ultra high performance liquid chromatography coupled with Fourier transform ion cyclotron resonance mass spectrometry. J Chromatogr B Analyt Technol Biomed Life Sci. 2018;1079:69-84.

12. Ding Z, Zhong R, Yang Y, et al: Systems pharmacology reveals the mechanism of activity of Ge-GenQin-Lian decoction against LPS-induced acute lung injury: A novel strategy for exploring active components and effective mechanism of TCM formulae. Pharmacol Res. 2020:104759.

13. Zhao Y, Luan H, Gao H, et al. Gegen Qinlian decoction maintains colonic mucosal homeostasis in acute/chronic ulcerative colitis via bidirectionally modulating dysregulated Notch signaling. 
Phytomedicine. 2020;68:153182.

14. W YF. Y, H H, et al: Efficacy of herbal medicine (Gegen Qinlian Decoction) on ulcerative colitis: A systematic review of randomized controlled trials. Medicine. 2019;98:e18512.

15. Yang J, Tian S, Zhao J, et al. Exploring the mechanism of TCM formulae in the treatment of different types of coronary heart disease by network pharmacology and machining learning. Pharmacol Res. 2020;159:105034.

16. Li S, Zhang B. Traditional Chinese medicine network pharmacology: theory, methodology and application. Chin J Nat Med. 2013;11:110-20.

17. Liu Y, Xue Q, Li A, et al. Mechanisms exploration of herbal pair of HuangQi-DanShen on cerebral ischemia based on metabonomics and network pharmacology. J Ethnopharmacol. 2020;253:112688.

18. Ru J, Li P, Wang J, et al. TCMSP: a database of systems pharmacology for drug discovery from herbal medicines. J Cheminform. 2014;6:13.

19. Lipinski CA, Lombardo F, Dominy BW, et al. Experimental and computational approaches to estimate solubility and permeability in drug discovery and development settings. Adv Drug Deliv Rev. 2001;46:3-26.

20. Tao W, Xu X, Wang X, et al. Network pharmacology-based prediction of the active ingredients and potential targets of Chinese herbal Radix Curcumae formula for application to cardiovascular disease. J Ethnopharmacol. 2013;145:1-10.

21. Xu X, Zhang W, Huang C, et al. A novel chemometric method for the prediction of human oral bioavailability. Int J Mol Sci. 2012;13:6964-82.

22. Piñero J, Bravo À, Queralt-Rosinach N, et al. DisGeNET: a comprehensive platform integrating information on human disease-associated genes and variants. Nucleic Acids Res. 2017;45:D833-d9.

23. Piñero J, Ramírez-Anguita JM, Saüch-Pitarch J, et al. The DisGeNET knowledge platform for disease genomics: 2019 update. Nucleic Acids Res. 2020;48:D845-d55.

24. Rebhan M, Chalifa-Caspi V, Prilusky J, et al. GeneCards: integrating information about genes, proteins and diseases. Trends Genet. 1997;13:163.

25. Fishilevich S, Zimmerman S, Kohn A, et al: Genic insights from integrated human proteomics in GeneCards. Database (Oxford). 2016; 2016.

26. Wishart DS, Feunang YD, Guo AC, et al. DrugBank 5.0: a major update to the DrugBank database for 2018. Nucleic Acids Res. 2018;46:D1074-d82.

27. Yu G, Wang LG, Han Y, et al. clusterProfiler: an R package for comparing biological themes among gene clusters. OMICS. 2012;16:284-7.

28. Scott DE, Bayly AR, Abell C, et al. Small molecules, big targets: drug discovery faces the proteinprotein interaction challenge. Nat Rev Drug Discov. 2016;15:533-50.

29. Szklarczyk D, Gable AL, Lyon D, et al. STRING v11: protein-protein association networks with increased coverage, supporting functional discovery in genome-wide experimental datasets. Nucleic 
Acids Res. 2019;47:D607-d13.

30. Chin $\mathrm{CH}$, Chen $\mathrm{SH}, \mathrm{Wu} \mathrm{HH}$, et al. cytoHubba: identifying hub objects and sub-networks from complex interactome. BMC Syst Biol. 2014;8(Suppl 4):11.

31. Liu Y, Grimm M, Dai WT, et al. CB-Dock: a web server for cavity detection-guided protein-ligand blind docking. Acta Pharmacol Sin. 2020;41:138-44.

32. Cao SY, Ye SJ, Wang WW, et al. Progress in active compounds effective on ulcerative colitis from Chinese medicines. Chin J Nat Med. 2019;17:81-102.

33. Zhang $\mathrm{C}$, Jiang M, Lu A. Considerations of traditional Chinese medicine as adjunct therapy in the management of ulcerative colitis. Clin Rev Allergy Immunol. 2013;44:274-83.

34. Zhang R, Zhu X, Bai H, et al. Network Pharmacology Databases for Traditional Chinese Medicine: Review and Assessment. Front Pharmacol. 2019;10:123.

35. Bhattacharyya A, Chattopadhyay R, Mitra S, et al. Oxidative stress: an essential factor in the pathogenesis of gastrointestinal mucosal diseases. Physiol Rev. 2014;94:329-54.

36. de Barrios O, Sanchez-Moral L, Cortés M, et al: ZEB1 promotes inflammation and progression towards inflammation-driven carcinoma through repression of the DNA repair glycosylase MPG in epithelial cells. Gut. 2019.

37. Banan A, Choudhary S, Zhang Y, et al. Ethanol-induced barrier dysfunction and its prevention by growth factors in human intestinal monolayers: evidence for oxidative and cytoskeletal mechanisms. J Pharmacol Exp Ther. 1999;291:1075-85.

38. Amati L, Caradonna L, Leandro G, et al. Immune abnormalities and endotoxemia in patients with ulcerative colitis and in their first degree relatives: attempts at neutralizing endotoxin-mediated effects. Curr Pharm Des. 2003;9:1937-45.

39. Sperandeo P, Martorana AM, Polissi A. Lipopolysaccharide biogenesis and transport at the outer membrane of Gram-negative bacteria. Biochim Biophys Acta Mol Cell Biol Lipids. 2017;1862:145160.

40. Brooks D, Barr LC, Wiscombe S, et al: Human lipopolysaccharide models provide mechanistic and therapeutic insights into systemic and pulmonary inflammation. Eur Respir J. 2020.

41. Lin TL, Shu CC, Chen YM, et al. Like Cures Like: Pharmacological Activity of Anti-Inflammatory Lipopolysaccharides From Gut Microbiome. Front Pharmacol. 2020;11:554.

42. Iboshi Y, Nakamura K, Fukaura K, et al. Increased IL-17A/IL-17F expression ratio represents the key mucosal T helper/regulatory cell-related gene signature paralleling disease activity in ulcerative colitis. J Gastroenterol. 2017;52:315-26.

43. Ueno A, Jeffery L, Kobayashi T, et al. Th17 plasticity and its relevance to inflammatory bowel disease. J Autoimmun. 2018;87:38-49.

44. Lin JX, Leonard WJ. Fine-Tuning Cytokine Signals. Annu Rev Immunol. 2019;37:295-324.

45. Salas A, Hernandez-Rocha C, Duijvestein M, et al: JAK-STAT pathway targeting for the treatment of inflammatory bowel disease. Nat Rev Gastroenterol Hepatol. 2020. 
46. He J, Song Y, Li G, et al. Fbxw7 increases CCL2/7 in CX3CR1hi macrophages to promote intestinal inflammation. J Clin Invest. 2019;129:3877-93.

47. Parisinos CA, Serghiou S, Katsoulis M, et al: Variation in Interleukin 6 Receptor Gene Associates With Risk of Crohn's Disease and Ulcerative Colitis. Gastroenterology. 2018; 155:303-6 e2.

48. Vilgelm AE, Richmond A. Chemokines Modulate Immune Surveillance in Tumorigenesis, Metastasis, and Response to Immunotherapy. Front Immunol. 2019;10:333.

49. Ha H, Debnath B, Neamati N. Role of the CXCL8-CXCR1/2 Axis in Cancer and Inflammatory Diseases. Theranostics. 2017;7:1543-88.

50. Fisher RC, Bellamkonda K, Alex Molina L, et al. Disrupting Inflammation-Associated CXCL8-CXCR1 Signaling Inhibits Tumorigenicity Initiated by Sporadic- and Colitis-Colon Cancer Stem Cells. Neoplasia. 2019;21:269-81.

51. Pott J, Maloy KJ. Epithelial autophagy controls chronic colitis by reducing TNF-induced apoptosis. Autophagy. 2018;14:1460-1.

52. Franke A, Balschun T, Karlsen TH, et al. Sequence variants in IL10, ARPC2 and multiple other loci contribute to ulcerative colitis susceptibility. Nat Genet. 2008;40:1319-23.

53. Esplugues E, Huber S, Gagliani N, et al. Control of TH17 cells occurs in the small intestine. Nature. 2011;475:514-8.

54. Wynn TA. Type 2 cytokines: mechanisms and therapeutic strategies. Nat Rev Immunol. 2015;15:271-82.

55. Rückert Y, Schindler U, Heinig T, et al. IL-4 Signaling Mechanisms in Inflammatory Bowel Disease Mononuclear Phagocytes. Inflamm Bowel Dis. 1996;2:244-52.

56. Tu L, Chen J, Zhang H, et al. Interleukin-4 Inhibits Regulatory T Cell Differentiation through Regulating CD103 + Dendritic Cells. Front Immunol. 2017;8:214.

57. Jayme TS, Leung G, Wang A, et al. Human interleukin-4-treated regulatory macrophages promote epithelial wound healing and reduce colitis in a mouse model. Sci Adv. 2020;6:eaba4376.

58. Malek TR. The biology of interleukin-2. Annu Rev Immunol. 2008;26:453-79.

59. Fontenot JD, Rasmussen JP, Gavin MA, et al. A function for interleukin 2 in Foxp3-expressing regulatory T cells. Nat Immunol. 2005;6:1142-51.

60. Lee H, Son YS, Lee MO, et al. Low-dose interleukin-2 alleviates dextran sodium sulfate-induced colitis in mice by recovering intestinal integrity and inhibiting AKT-dependent pathways. Theranostics. 2020;10:5048-63.

61. Musso A, Dentelli P, Carlino A, et al. Signal transducers and activators of transcription 3 signaling pathway: an essential mediator of inflammatory bowel disease and other forms of intestinal inflammation. Inflamm Bowel Dis. 2005;11:91-8.

62. Mudter J, Weigmann B, Bartsch B, et al. Activation pattern of signal transducers and activators of transcription (STAT) factors in inflammatory bowel diseases. Am J Gastroenterol. 2005;100:64-72. 
63. Durant L, Watford WT, Ramos HL, et al. Diverse targets of the transcription factor STAT3 contribute to T cell pathogenicity and homeostasis. Immunity. 2010;32:605-15.

64. Jairath V, Khanna R, Feagan BG. Alicaforsen for the treatment of inflammatory bowel disease. Expert Opin Investig Drugs. 2017;26:991-7.

65. Dustin ML, Bivona TG, Philips MR. Membranes as messengers in T cell adhesion signaling. Nat Immunol. 2004;5:363-72.

66. Watanabe T, Fan J. Atherosclerosis and inflammation mononuclear cell recruitment and adhesion molecules with reference to the implication of ICAM-1/LFA-1 pathway in atherogenesis. Int $\mathrm{J}$ Cardiol. 1998;66(Suppl 1):45-53. discussion S5.

67. Etienne-Manneville S, Chaverot N, Strosberg AD, et al. ICAM-1-coupled signaling pathways in astrocytes converge to cyclic AMP response element-binding protein phosphorylation and TNF-alpha secretion. J Immunol. 1999;163:668-74.

68. Reyes-Farias M, Carrasco-Pozo C. The Anti-Cancer Effect of Quercetin: Molecular Implications in Cancer Metabolism. Int J Mol Sci. 2019; 20.

69. McKay TB, Karamichos D. Quercetin and the ocular surface: What we know and where we are going. Exp Biol Med (Maywood). 2017;242:565-72.

70. Carullo G, Cappello AR, Frattaruolo L, et al. Quercetin and derivatives: useful tools in inflammation and pain management. Future Med Chem. 2017;9:79-93.

71. Dicarlo M, Teti G, Verna G, et al: Quercetin Exposure Suppresses the Inflammatory Pathway in Intestinal Organoids from Winnie Mice. Int J Mol Sci. 2019; 20.

72. Sánchez de Medina F, Vera B, Gálvez J, et al. Effect of quercitrin on the early stages of hapten induced colonic inflammation in the rat. Life Sci. 2002;70:3097-108.

73. Dey P. Gut microbiota in phytopharmacology: A comprehensive overview of concepts, reciprocal interactions, biotransformations and mode of actions. Pharmacol Res. 2019;147:104367.

74. Nie J, Zhang L, Zhao G, et al. Quercetin reduces atherosclerotic lesions by altering the gut microbiota and reducing atherogenic lipid metabolites. J Appl Microbiol. 2019;127:1824-34.

75. Guimbaud R, Bertrand V, Chauvelot-Moachon L, et al. Network of inflammatory cytokines and correlation with disease activity in ulcerative colitis. Am J Gastroenterol. 1998;93:2397-404.

76. Abraham C, Dulai PS, Vermeire S, et al: Lessons Learned From Trials Targeting Cytokine Pathways in Patients With Inflammatory Bowel Diseases. Gastroenterology. 2017; 152:374 - 88.e4.

\section{Figures}




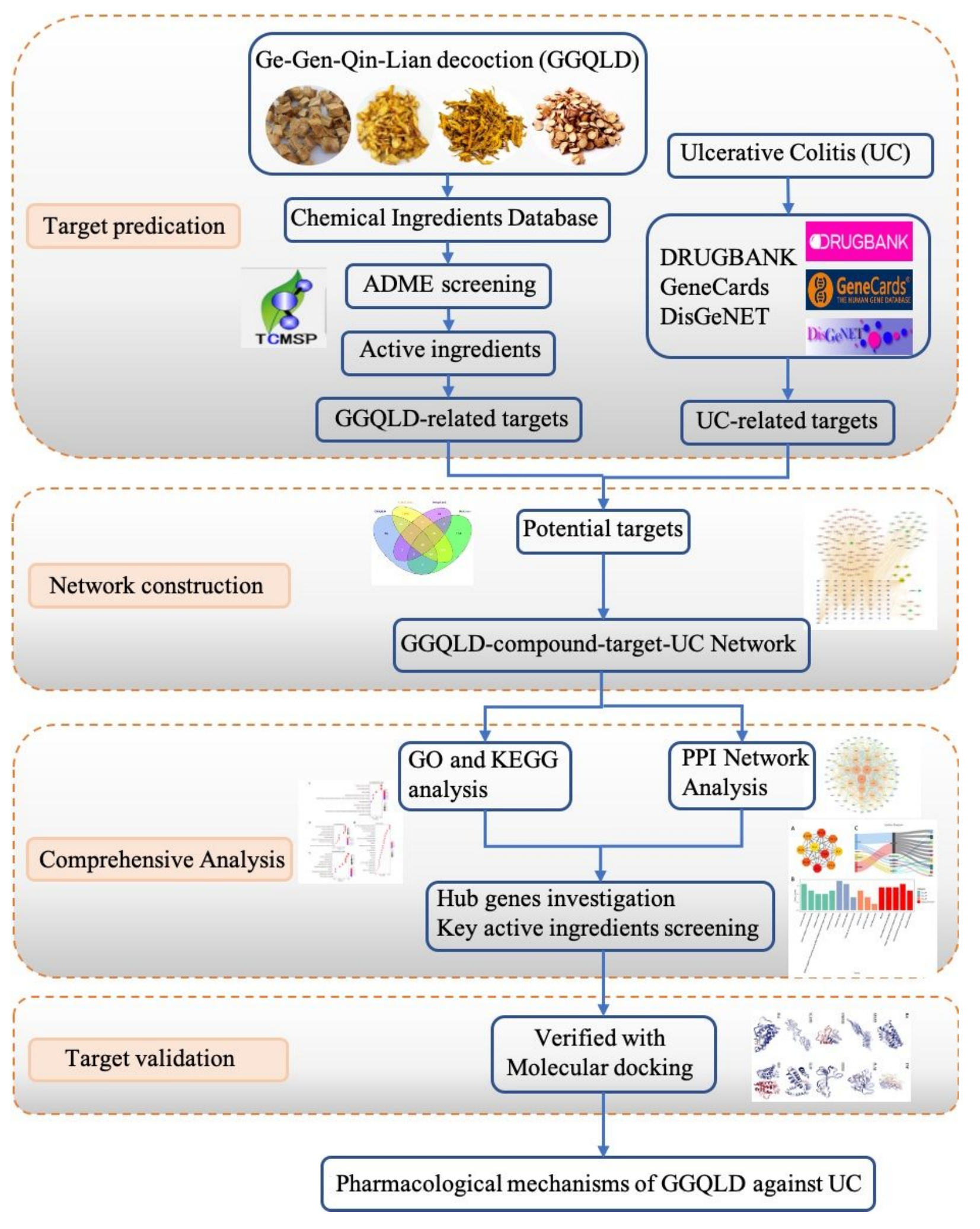

\section{Figure 1}

The graphical abstract for underlining Pharmacological mechanisms of GGQLD against UC. 


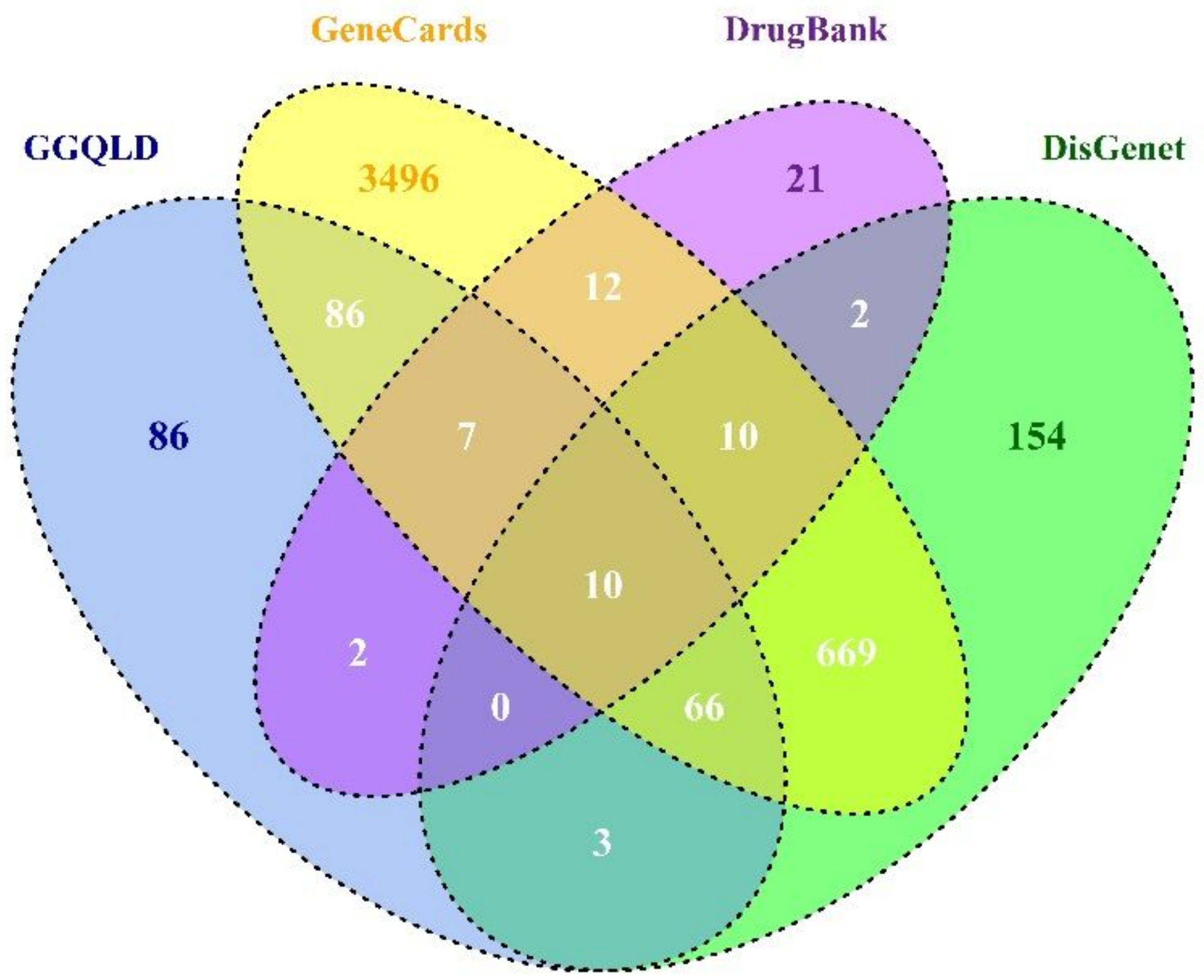

Figure 2

Venn diagram. Venn diagram showing the numbers of the overlapped and the specific genes among the four databases (GGQLD, DisGenet, GeneCards, DrugBank). The overlapping genes were selected for further analysis. 


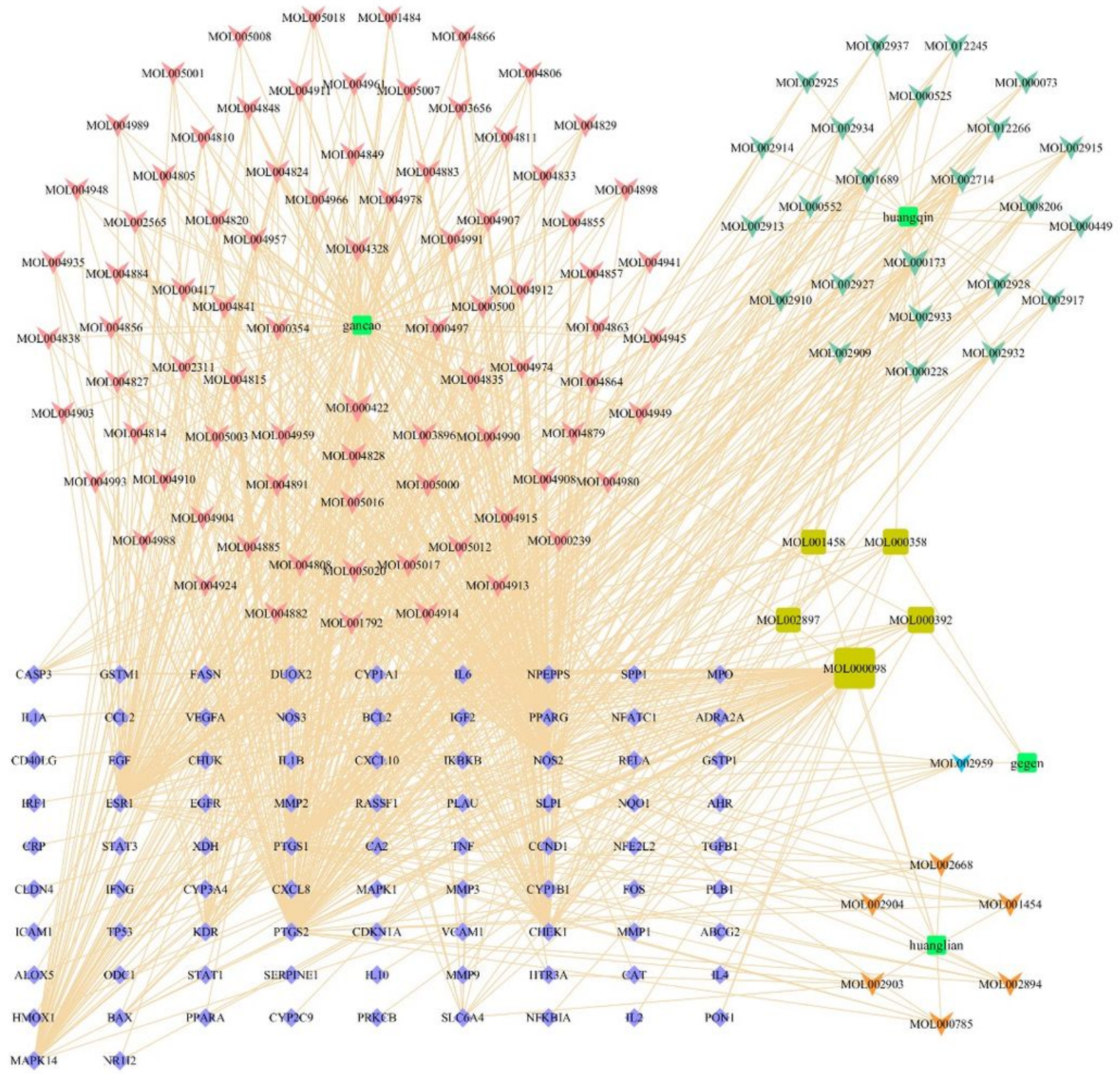

Figure 3

The G-U network. The green square represented the drug; the purple diamond represented the potential targets; the arrow represented the drug ingredients, color means different herbal; the olive square means shared ingredients. The line between two nodes represented the interaction, the size of each node indicates the number of connections. 
A

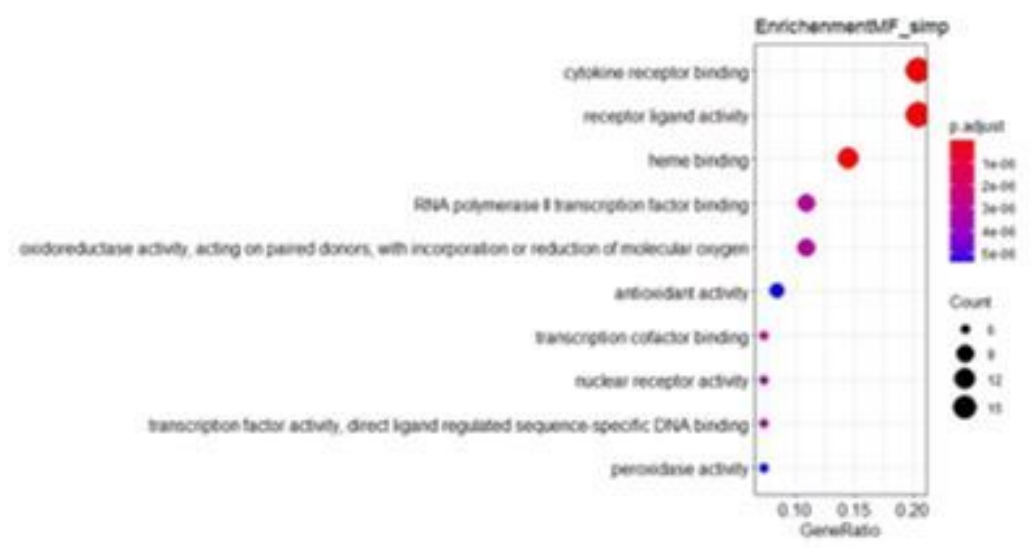

\section{B}

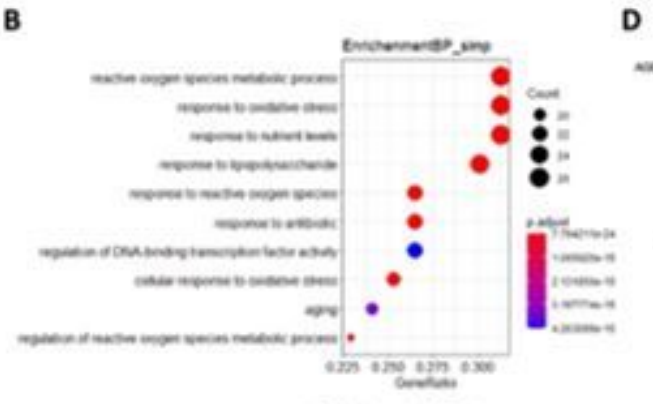

C
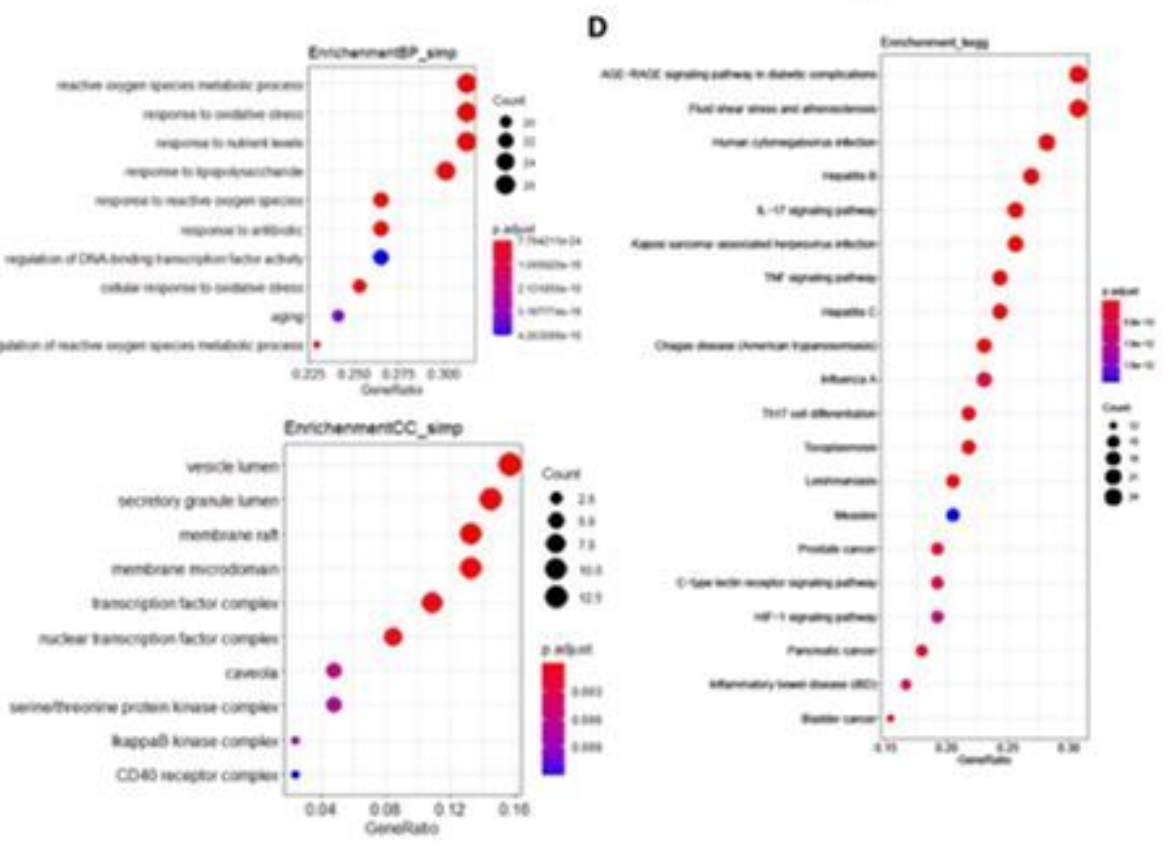

\section{Figure 4}

GO and KEGG pathway enrichment analysis result. (A) MF enrichment analysis; (B) BP enrichment analysis; (C) CC enrichment analysis; (D) KEGG enrichment analysis. The size of each node indicates enriched counts, the abscissa represents the enriched gene ratio, color means enriched adjust P-value. 


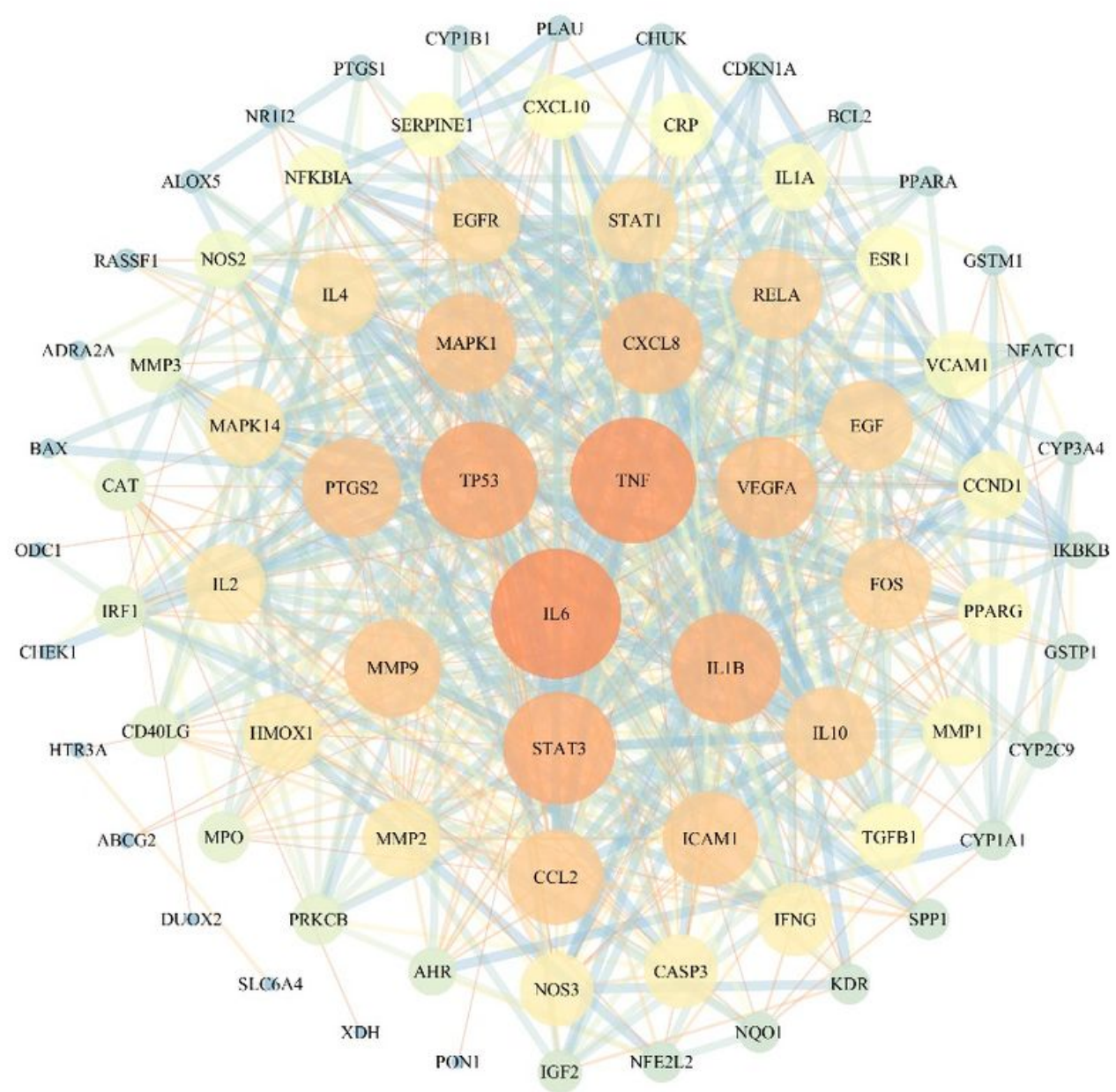

Figure 5

The PPI network of the potential targets. Node size from large to small represents the descending order of the degree value. The line width between two nodes represented the interaction, the darker the color, the more significant it is. 


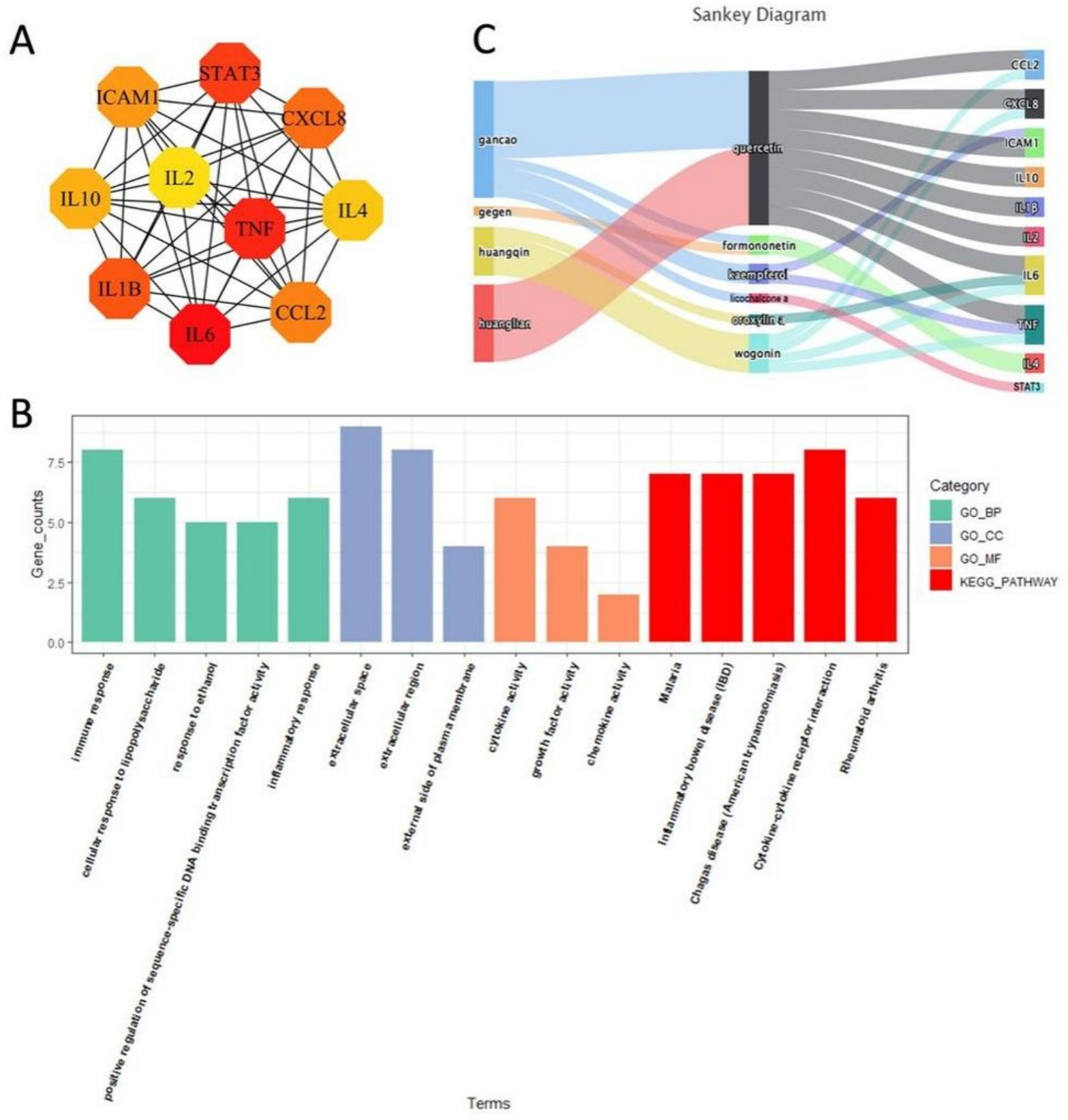

Figure 6

The result of hub genes investigation and screening of key active ingredients. (A) The 10 hub genes identified from the PPI network. the darker the color, the more significant it is. (B) The top $5 \mathrm{GO}$ terms and KEGG pathways of the 10 hub genes. Each bar indicates a BP/CC/MF/KEGG term, color represents an enriched type, abscissa means the number of enriched genes. (C) Sankey diagram. Sankey diagram reveals the relationship between herb, ingredients, and targets. The left blocks represent the herb, the middle blocks represent the ingredients, the right blocks represent the hub targets. 
$\mathbf{A}$

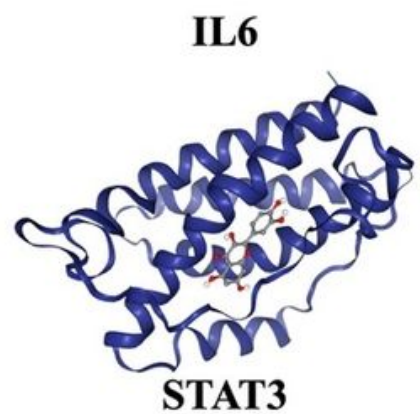

C

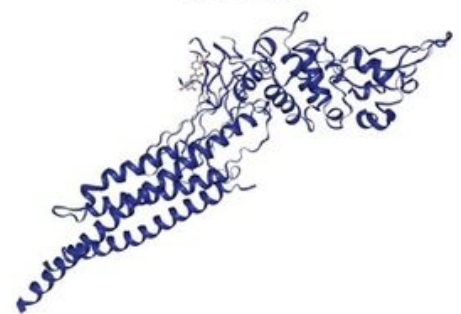

$\mathbf{E}$

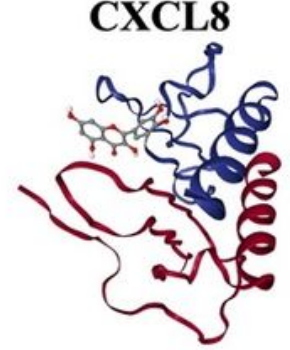

G

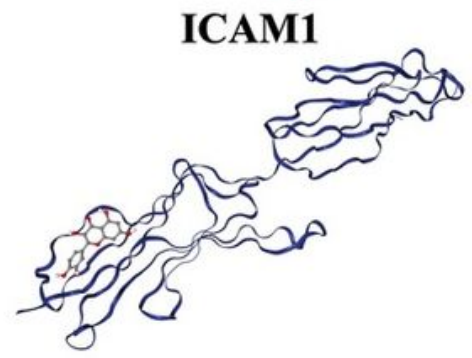

I

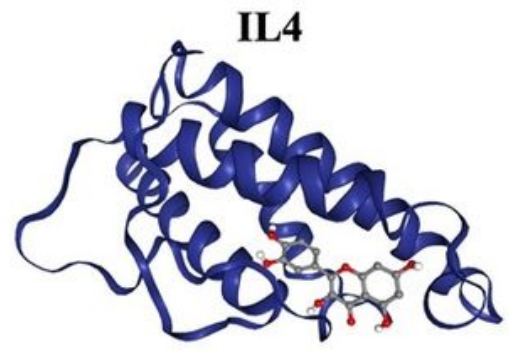

B

TNF

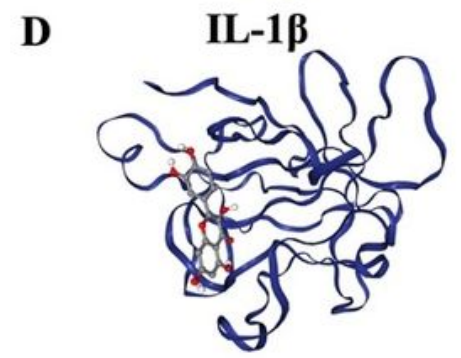

$\mathbf{F}$

CCL2

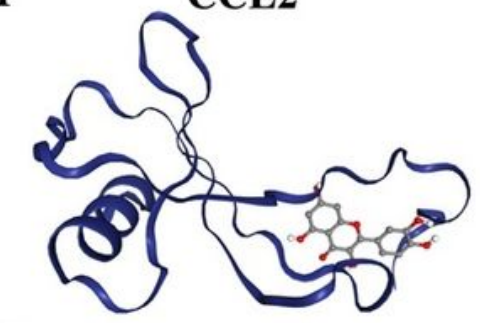

$\mathbf{H}$
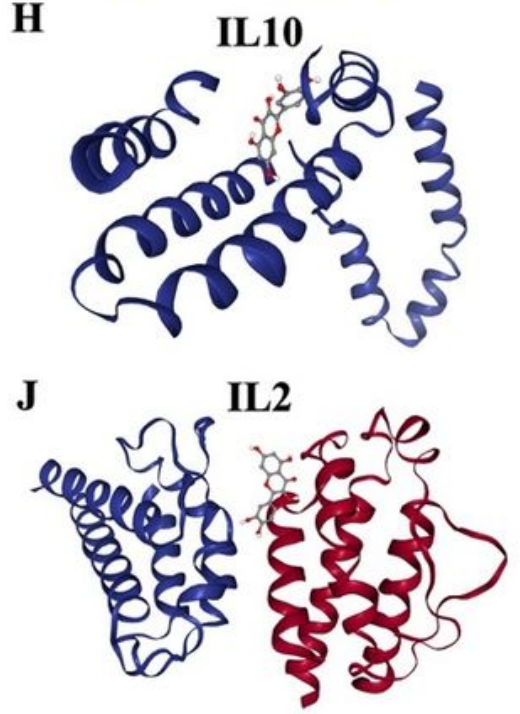

Figure 7

Molecular docking models of quercetin binding to the 10 hub targets (IL6 (A), TNF (B), STAT3 (C), IL1 $\beta$ (D), CXCL8 (E), CCL2 (F), ICAM1 (G), IL10 (H), IL4 (I), IL2 (J)). Ball\&stick represents quercetin; cartoon represents a hub target.

\section{Supplementary Files}


This is a list of supplementary files associated with this preprint. Click to download.

- tableS5GOandKEGGenrichmentofthe10hubgenes.pdf

- tableS4GOandKEGGAnalysis.pdf

- tableS3GGQLDcompoundtargetUCNetwork.pdf

- tableS2targetinformation.pdf

- tableS1BioactiveCompoundsinGGQLD.pdf 